\title{
Difficulties of Subsurface Liquid Front Tracking in Porous Media with Terahertz Pulsed Imaging
}

\author{
M. Al-Sharabi ${ }^{1}$, T. Mudley ${ }^{1}$, D. Markl ${ }^{2}$, P. Bawuah ${ }^{1}$, A-P. Karttunen ${ }^{3}$, C. J. Ridgway ${ }^{4}$, P.A.C. Gane ${ }^{4,5}$, \\ J. Ketolainen ${ }^{3}$, K-E. Peiponen ${ }^{6}$, T. Rades ${ }^{7}$ and J. A. Zeitler ${ }^{1}$ \\ ${ }^{1}$ Department of Chemical Engineering and Biotechnology, University of Cambridge, Cambridge, CB3 0AS, UK \\ ${ }^{2}$ Strathclyde Institute of Pharmacy and Biomedical Sciences, University of Strathclyde, G4 ORE Glasgow, UK \\ ${ }^{3}$ School of Pharmacy, Promis Centre, University of Eastern Finland, FI-70211 Kuopio, Finland \\ ${ }^{4}$ Omya International AG, Baslerstrasse 42, CH-4665 Oftringen, Switzerland \\ ${ }^{5}$ Aalto University, Department of Bioproducts and Biosystems, FI-00076 Aalto, Finland \\ ${ }^{6}$ Institute of Photonics, University of Eastern Finland, FI-80101 Joensuu, Finland \\ ${ }^{7}$ Department of Pharmacy, University of Copenhagen, Universitetsparken 2, DK-2100 Copenhagen, Denmark
}

\begin{abstract}
Terahertz pulsed imaging (TPI) coupled with a customised flow cell has previously been used successfully to study liquid transport in porous media, such as powder compacts. In some samples, the tracking of the liquid front remains challenging when the refractive indices of liquid and sample are similar and hence little contrast is observed. In this work, we found that even when the relative difference between the refractive indices of liquid and sample is high, it can still be challenging to track the reflection peak of water in some samples while it works fine in others. It is hypothesised that this is due to the formation of a water gradient in these samples due to their specific microstructure and we explore methods to overcome this limitation.
\end{abstract}

\section{INTRODUCTION}

$\mathrm{T}$ ERAHERTZ pulsed imaging (TPI) has been used successfully to study the liquid transport kinetics of powder compacts $[1,2]$. The method exploits the difference between the refractive indices of the dry material and liquid, which results in a reflection of the terahertz pulse from the interface between the two media and therefore enables the tracking of the liquid front within the porous material [3]. Challenges arise when the refractive indices of the sample and penetrating liquid are similar. The amplitude of the reflection at the interface is governed by the difference in the refractive indices of the two media the pulse is propagating through [3]. However, in this work, the small amplitude of the liquid front reflection cannot be attributed to the similarity in the refractive indices of the liquid (water, $n \approx 2.1$ at $1 \mathrm{THz}$ ) and sample (functionalised calcium carbonate (FCC) based formulation, $n$ $\approx 2$ and microcrystalline cellulose (MCC) based formulation, $n$ $\approx 1.76$ ) as it was possible to resolve it for a smaller relative difference (Figure 1a).

\section{METHODS}

TPI in a reflection setup in combination with a flow cell was used to study the one-directional liquid ingress into tablets as described previously [3]. The acquired TPI data were first deconvolved in order to remove noise and enhance the contrast of the reflection peaks of the terahertz pulse, particularly the reflection peak of the water front [1]. The deconvolution is performed by dividing the raw sample signal by the reference signal in the frequency-domain and using a double Gaussian band-pass filter [3].

\section{RESULTS}

Theoretically, a larger difference in the refractive indices should result in a sharp liquid penetration front peak, which can be identified and tracked easily. Therefore, it is expected that the amplitude of the water peak reflection should be higher for the MCC containing tablets due to the higher relative difference between the refractive indices of the water and the MCC containing tablets in comparison to the FCC containing tablets. Based on Fresnel's theory [3], the reflection coefficient is $r_{12}=$ 0.02. and $r_{12}=0.09$. for the FCC and for MCC based formulations, respectively. Given the reflection amplitude from a mirror was 1.5 a.u., this should result in an amplitude of 0.03 a.u. (FCC based formulation) and 0.14 a.u. (MCC based formulation), but we found an average amplitude of 0.06 a.u. and 0.04 a.u., respectively, instead. a)

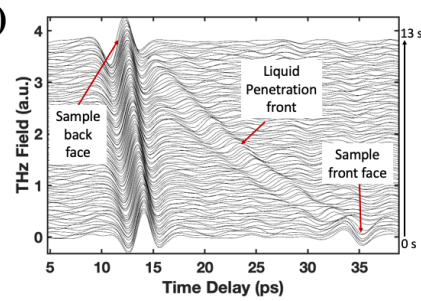

b)

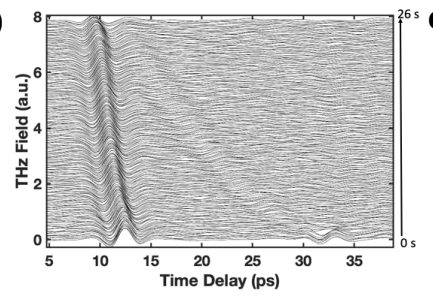

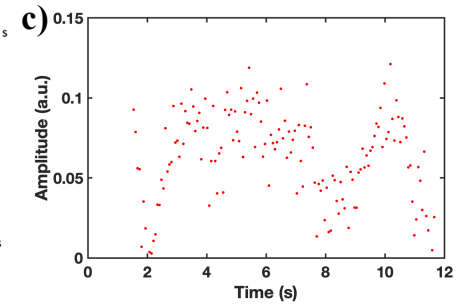

d)

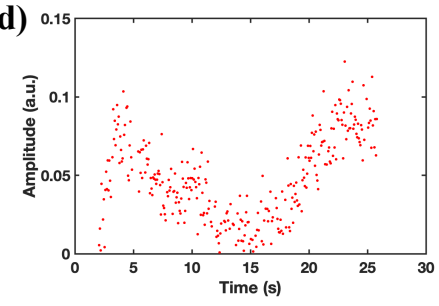

Figure 1. Analysis of the liquid fron tracking using TPI. (a,b) The waterfall plots and (c,d) the amplitude of the water front reflection as a function of time for two different samples with a relative refractive index change between liquid and sample of 0.1 and 0.34 for a) FCC and b) MCC based samples, respectively.

FCC has a large consistent pore space since its intra-particle pore space does not change during the compaction process. This pore space might be the main driving factor for the liquid ingress into the tablet rather than the inter-particle pore space and therefore a water gradient is not encountered in the FCC based tablets. In the case of the MCC based tablets, the inter- 
particle pore space acts as the predominant driving factor for the liquid penetration into the tablet matrix. This inter-particle pore space is not uniform within the tablet as a density gradient is formed across the tablet during compaction. Therefore, it can be hypothesised that the weak reflection of the water front in the MCC based tablets in Figure 1b (compared to Figure 1a) is due to the existence of microchannels which promote capillary action resulting in the wetting of the dry material before the bulk of the liquid has reached it. This introduces a water gradient into the sample. This will directly decrease the amplitude of the reflected terahertz pulse. Figure 1d shows that the amplitude of the water front reflection changes as the water penetrates through the MCC based sample due to a change of the refractive index of the material and hence results in a poor contrast in regions with similar refractive indices to that of water.

\section{CONCLUSIONS}

In conclusion, we observe for some type of porous materials a step change in the refractive index due to a well-defined water front propagating through the dry matrix (Figure 2, left). In contrast, for other materials a gradient in the refractive index is observed in the terahertz measurements causing a wetting distribution at the liquid penetration front. Whether or not a gradient or a step change is observed is dependent on the microstructure and physico-chemical properties of the materials in question. FCC is a rigid ceramic material and hence no swelling occurs upon hydration, whilst for $\mathrm{MCC}$, a polymer, swelling will take place concurrently with hydration resulting in more complex liquid transport behaviour that can be resolved by the terahertz method. Further work needs to be done to provide solutions to overcome the limitation of the poor contrast and optimise the data processing for a better peak tracking of the liquid front.
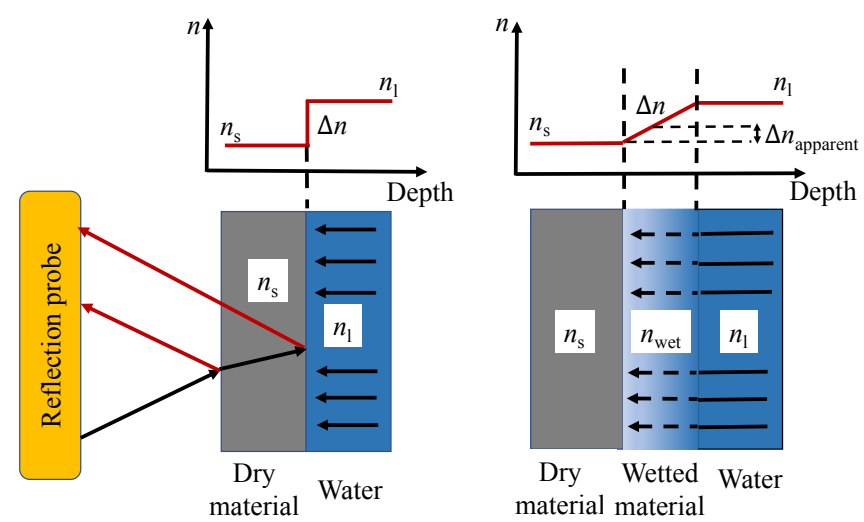

Figure 2. A schematic showing the impact of the water gradient on the relative difference of the refractive indices hence the amplitude of the water front reflection peak.

\section{ACKNOWLEDGEMENTS}

The authors would like to thank Johnson Matthey and the EPSRC for their funding, and Omya International AG for providing the FCC and experimental surface area and porosimetric analyses.

\section{REFERENCES}

[1] S. Yassin, K. Su, H. Lin, L. F. Gladden, J. A. Zeitler, "Diffusion and swelling measurements in pharmaceutical powder compacts using terahertz pulsed imaging," Journal of Pharmaceutical Sciences, vol.104, pp. 1658-1667, 2015.

[2] S. Yassin, D. J. Goodwin, A. Anderson, J. Sibik, D. I. Wilson, L. F. Gladden, J. A. Zeitler, "The Disintegration process in micro crystalline cellulose based tablets, part 1: Influence of temperature, porosity and superdisintegrants," Journal of Pharmaceutical Sciences, vol.104, pp. 3440-3450, 2015.

[3] D. Mark1, P. Wang, C. Ridgway, A.-P. Karttunen, P. Bawuah, J. Ketolainen, P. Gane, K.-E. Peiponen, J. A. Zeitler, "Resolving the rapid water absorption of porous functionalised calcium carbonate powder compacts by terahertz pulsed imaging," Chemical Engineering Research and Design, vol. 132, pp. 1082-1090, 2018. 